\title{
Imaging inflammation in atherosclerotic plaques: Just make it easy!
}

\author{
Fabien Hyafil, MD, PhD, ${ }^{\mathrm{a}}$ and Jonathan Vigne, PharmD ${ }^{\mathrm{a}}$ \\ a Department of Nuclear Medicine, Centre Hospitalier Universitaire Bichat, Assistance Publique - \\ Hôpitaux de Paris, Département Hospitalo-Universitaire FIRE, Inserm 1148, Université Paris \\ Diderot, Paris, France
}

Received Apr 14, 2018; accepted Apr 16, 2018

doi: 10.1007/s12350-018-1289-5

The presence of inflammatory cells is a hallmark of unstable atherosclerotic plaques. Several imaging approaches have been developed for the noninvasive detection of inflammatory activities in atherosclerotic plaques. Positron emission tomography (PET) imaging with the injection of 18F-fluorodeoxyglucose (FDG) is currently the most widely used imaging technique to evaluate the density of activated macrophages in atherosclerotic plaques. Nevertheless, FDGPET imaging has logistical and technical constraints that represent an important obstacle to the wider use of this approach for the evaluation of patients with atherosclerosis. In a similar way as in the oncological field, the balance between the benefits and costs of new drugs need to be improved in patients with cardiovascular diseases. PET imaging of plaque inflammation might represent a very useful tool to identify patients who could benefit the most from anti-inflammatory treatments and to exclude patients with other causes of inflammation who are the most likely to develop severe side effects under these drugs. The availability of radiotracers targeting more specifically inflammation in atherosclerotic plaques would greatly facilitate the logistic organization of this imaging and help to expand the use of PET for the evaluation of atherosclerotic patients.

Key Words: Atherosclerosis • PET • molecular imaging • vascular imaging • inflammation • molecular imaging agents

\section{See related article, pp. 1697-1704}

\section{ROLE OF INFLAMMATION IN PLAQUE DESTABILIZATION}

The presence of inflammatory cells is a hallmark of unstable atherosclerotic plaques. Ruptured atherosclerotic plaques contain higher densities of macrophages

\footnotetext{
Reprint requests: Fabien Hyafil, MD, PhD, Department of Nuclear Medicine, Centre Hospitalier Universitaire Bichat, Assistance Publique - Hôpitaux de Paris, Département Hospitalo-Universitaire FIRE, Inserm 1148, Université Paris Diderot, 46 rue Henri Huchard, 75018 Paris, France; fabien.hyafil@aphp.fr

J Nucl Cardiol 2019;26:1705-8.

$1071-3581 / \$ 34.00$

Copyright (c) 2018 American Society of Nuclear Cardiology.
}

and lymphocytes ${ }^{1}$ than plaques from patients with stable coronary artery disease (CAD). Macrophages play a key role in plaque destabilization: they release locally pro-inflammatory cytokines and secrete enzymes capable of directly digesting the fibrous cap of the plaque. Where the degree of inflammation is sufficient, the fibrous cap can rupture, exposing the thrombogenic lipid core to the bloodstream. This may cause local arterial thrombosis and result in clinical events such as myocardial infarction or ischemic stroke. The infiltration of atherosclerotic plaques by inflammatory cells is not limited to the culprit lesion in patients dying from acute coronary syndromes, but has also been observed in all atherosclerotic plaques present along the coronary arterial beds in favor of a diffuse inflammatory process in the vessel wall of symptomatic patients. ${ }^{2}$ The intensity of inflammation in atherosclerotic plaques can be approached by measuring the level of high-sensitivity $\mathrm{C}$ reactive protein (hs-CRP) in blood. Elevated levels of hs-CRP have been associated with an increased risk of cardiovascular events independent of the cholesterol 
level. ${ }^{3,4}$ A limitation of hs-CRP is its lack of specificity for inflammation in atherosclerotic plaques. In particular, myocardial or cerebral necrosis following plaque rupture and arterial thrombosis can cause an increase in CRP values that limits its value for grading the intensity of plaque inflammation in patients with acute ischemic complications of atherosclerosis. Based on the results of observational studies showing the increased risk of patients with stable atherosclerosis and high hs-CRP values, interventional clinical studies have been set up to evaluate whether anti-inflammatory drugs might be effective to prevent cardiovascular events. The Canakinumab Antiinflammatory Thrombosis Outcome Study (CANTOS) study ${ }^{5}$ has tested the efficacy of canakinumab, an inhibitor of interleukin- $1 \beta$, in 10000 patients with coronary heart disease and hs-CRP $>2 \mathrm{mg} / \mathrm{L}$. At 4 years, a small decrease in the rate of cardiovascular events was evidenced in patients treated with the inhibitor of interleukin- $1 \beta$, but at the expense of an increased incidence of severe infections. The results of the CANTOS study underscore the need for specific biomarkers of vascular inflammation to identify atherosclerotic patients who might benefit the most from anti-inflammatory treatments.

\section{IMAGING INFLAMMATION WITH PET}

In the past 20 years, several imaging technologies have been developed for the noninvasive detection of inflammatory activities in atherosclerotic plaques. ${ }^{6}$ Positron emission tomography (PET) imaging with the injection of 18F-fluorodeoxyglucose (FDG) is currently the most widely used imaging technique to evaluate the density of activated macrophages in atherosclerotic plaques. FDG is avidly taken up by activated hypoxic macrophages and its uptake in vessel wall correlated closely with the density of macrophages determined histologically both in animal models of atherosclerosis and in patients with carotid disease. ${ }^{7,8}$ Nevertheless, FDG-PET imaging has logistical and technical constraints that represent an important obstacle to a wider use of this approach for the evaluation of patients with atherosclerosis. For FDG-PET acquisition, patients need to fast at least $6 \mathrm{~h}$ before the imaging to limit FDG uptake in peripheral muscle and maintain blood glucose in normal ranges. In addition, myocardial FDG uptake should be suppressed for the analysis of coronary arteries by extending the fasting period to $12 \mathrm{~h}$ and asking patients to start a low-carb high-fat diet $24 \mathrm{~h}$ before PET acquisition. ${ }^{9}$ In carotid arteries, the analysis of the vascular signal can be hampered by the high FDG uptake in structures in close vicinity to the vessels such as lymph nodes or cervical muscles. Radiotracers that target more specifically inflammatory cells in the vessel wall would greatly facilitate the noninvasive evaluation of atherosclerosis with PET.

\section{RADIOTRACERS ALTERNATIVE TO FDG FOR THE DETECTION OF INFLAMMATION IN ATHEROSCLEROTIC PLAQUES}

In this issue of the Journal of Nuclear Cardiology, Meester EJ et al. ${ }^{10}$ have tested the tracer DOTAbutylamino-NorBIRT (DANBIRT) labeled with ${ }^{111}$ Indium in a mice model of atherosclerosis. DANBIRT binds to Leukocyte Function-associated Antigen-1 (LFA-1) expressed on leukocytes and might thus represent an interesting candidate tracer for the more specific identification on inflammatory activities in atherosclerotic plaques. The long half-life of ${ }^{111}$ Indium associated to its low SPECT signal and high radiation burden represent an important limitation of this approach. Nevertheless, ${ }^{111}$ Indium can be replaced by ${ }^{68}$ Gallium in the DOTA cycle resulting in a PET radiotracer that might have favorable properties for atherosclerosis imaging. DANBIRT would then extend the list of radiotracers that target receptors expressed on inflammatory cells in atherosclerotic plaques, which already include the somatostatin receptor SST $2,{ }^{11}$ the translocator protein $\mathrm{TSPO},{ }^{12}$ the mannose receptor, ${ }^{13}$ CXCR $4,{ }^{14}$ the folate ${ }^{15}$ and interleukin receptors, ${ }^{16}$ and the choline transporter. ${ }^{17}$ One might wonder why none of these radiotracers has managed until now to effectively replace FDG for the evaluation of inflammation in atherosclerotic plaques. In fact, one of the challenges in detecting inflammation in plaque is that this activity is concentrated in a relatively small area of the vascular wall, the region surrounding the lipid core including the fibrous cap with some signal also extending in the adjacent adventitia, in contrast to active vasculitis in which a large number of inflammatory cells infiltrate the entire vascular wall. FDG presents the advantage of providing a strong signal on PET thanks to its high accumulation in metabolically active cells and to the expression of GLUT receptors on a large variety of inflammatory cells, including activated macrophages and lymphocytes. Novel radiotracers have been developed as alternative to FDG to detect specifically activated macrophages. The challenge for these radiotracers is to target a molecule or biological activity that is sufficiently expressed in atherosclerotic plaques to be detectable on PET, and also to demonstrate that this target is clinically relevant for the evaluation of atherosclerosis in patients, i.e., an increase in the PET signal is associated with the progression of atherosclerosis and the risk of cardiovascular events. In addition to 
scientific aspects, the business model to bring on the market a radiotracer dedicated only to atherosclerosis imaging is currently complex and risky for radiopharmaceutical companies. A more realistic approach is thus to develop radiotracers targeting inflammatory cells with clinical applications in other pathologies than atherosclerosis, or to identify radiotracers targeting receptors expressed on tumor cells that are also present on activated macrophages. Using the latter strategy, two PET radiotracers, ${ }^{68} \mathrm{Ga}$-DOTATATE and ${ }^{68} \mathrm{Ga}$-pentixafor, have recently been found to accumulate in atherosclerotic plaques and to bind, respectively, to the SSTR2 and CXCR4 receptors expressed on activated macrophages. Tarkin et al. ${ }^{18}$ tested ${ }^{68}$ Ga-DOTATATE for the detection of inflammation in atherosclerotic plaques. They found in patients imaged sequentially with FDG and ${ }^{68} \mathrm{Ga}$-DOTATATE a good correlation between the intensity of the signal measured in coronary and carotid atherosclerotic plaques between the two radiotracers, and improved image quality with ${ }^{68} \mathrm{Ga}$ DOTATATE thanks to less background signal in structures adjacent to plaques in comparison to FDG. PET radiotracers targeting SST2 present the advantage that they have already approved for the evaluation of patients with neuro-endocrine tumors. This should greatly facilitate the evaluation of these radiotracers for the detection of inflammation in atherosclerotic plaques.

\section{CONCLUSION}

In the last years, major developments in noninvasive imaging technologies of the vessels have allowed us to move from a view centered on the degree of luminal stenosis to a broader vision showing the variety and complexity of atherosclerotic plaques that develop in the vessel wall over time. Patients with similar degrees of luminal stenosis can have very different plaque aspects. Several morphological and molecular imaging biomarkers of high-risk atherosclerotic plaques have been identified and associated to an increased risk of cardiovascular events. In a similar way as in the oncological field, the balance between the benefits and costs of new drugs need to be improved in patients with cardiovascular diseases. Nevertheless, information on plaque composition provided by imaging is rarely used for the selection of patients for treatments. PET imaging of plaque inflammation might represent a very useful tool to identify patients who could benefit the most from anti-inflammatory treatments and to exclude patients with other causes of inflammation who are the most likely to develop severe side effects under these drugs. The availability of radiotracers targeting more specifically inflammation in atherosclerotic plaques would greatly simplify the logistic organization for this imaging approach and could help to expand the use of PET for the evaluation of atherosclerotic patients.

\section{Disclosure}

F Hyafil and J. Vigne have nothing to disclose in relation to this Editorial.

\section{References}

1. Naghavi M, Libby P, Falk E, et al. From vulnerable plaque to vulnerable patient: a call for new definitions and risk assessment strategies: part I. Circulation 2003;108:1664-1672.

2. Libby P, Ridker PM, Maseri A. Inflammation and atherosclerosis. Circulation 2002;105:1135-43.

3. Ridker PM, Cushman M, Stampfer MJ, Tracy RP, Hennekens CH. Inflammation, aspirin, and the risk of cardiovascular disease in apparently healthy men. N Engl J Med 1997;336:973-9.

4. Ridker PM, Rifai N, Pfeffer MA, et al. Inflammation, pravastatin, and the risk of coronary events after myocardial infarction in patients with average cholesterol levels. Cholesterol and Recurrent Events (CARE) Investigators. Circulation 1998;98:839-44.

5. Ridker PM, Everett BM, Thuren T, et al. Antiinflammatory therapy with canakinumab for atherosclerotic disease. N Engl J Med 2017;377:1119-31.

6. Rudd JH, Hyafil F, Fayad ZA. Inflammation imaging in atherosclerosis. Arterioscler Thromb Vasc Biol 2009;29:100916.

7. Hyafil F, Cornily JC, Rudd JH, Machac J, Feldman LJ, Fayad ZA. Quantification of inflammation within rabbit atherosclerotic plaques using the macrophage-specific CT contrast agent N1177: a comparison with 18F-FDG PET/CT and histology. J Nucl Med 2009;50:959-65.

8. Tawakol A, Migrino RQ, Bashian GG, et al. In vivo 18F-fluorodeoxyglucose positron emission tomography imaging provides a noninvasive measure of carotid plaque inflammation in patients. $\mathbf{J}$ Am Coll Cardiol 2006;48:1818-24.

9. Bucerius J, Hyafil F, Verberne HJ, et al. Position paper of the Cardiovascular Committee of the European Association of Nuclear Medicine (EANM) on PET imaging of atherosclerosis. Eur J Nucl Med Mol Imaging 2016;43:780-92.

10. Meester EJ, Krenning BJ, de Blois $\mathrm{RH}$, et al. Imaging of atherosclerosis, targeting LFA-1 on inflammatory cells with ${ }^{111} \mathrm{In}$ DANBIRT. J Nucl Cardiol 2018. https://doi.org/10.1007/s12350018-1244-5.

11. Pedersen SF, Sandholt BV, Keller SH, et al. 64Cu-DOTATATE PET/MRI for detection of activated macrophages in carotid atherosclerotic plaques: studies in patients undergoing endarterectomy. Arterioscler Thromb Vasc Biol 2015;35:1696703.

12. Gaemperli $\mathrm{O}$, Shalhoub $\mathrm{J}$, Owen $\mathrm{DR}$, et al. Imaging intraplaque inflammation in carotid atherosclerosis with 11C-PK11195 positron emission tomography/computed tomography. Eur Heart $\mathbf{J}$ 2012;33:1902-10

13. Varasteh Z, Hyafil F, Anizan N, et al. Targeting mannose receptor expression on macrophages in atherosclerotic plaques of apolipoprotein E-knockout mice using 111In-tilmanocept. EJNMMI Res 2017;7:40.

14. Hyafil F, Pelisek J, Laitinen I, et al. Imaging the cytokine receptor CXCR4 in atherosclerotic plaques with the radiotracer $68 \mathrm{Ga}-$ pentixafor for PET. J Nucl Med 2017;58:499-506. 
15. Jager NA, Westra J, Golestani R, et al. Folate receptor-beta imaging using $99 \mathrm{mTc}$-folate to explore distribution of polarized macrophage populations in human atherosclerotic plaque. J Nucl Med 2014;55:1945-51.

16. Di Gialleonardo V, Signore A, Glaudemans AW, Dierckx RA, De Vries EF. N-(4-18F-fluorobenzoyl)interleukin-2 for PET of human-activated T lymphocytes. J Nucl Med 2012;53:679-86.
17. Bucerius J, Schmaljohann J, Bohm I, et al. Feasibility of $18 \mathrm{~F}-$ fluoromethylcholine PET/CT for imaging of vessel wall alterations in humans-first results. Eur J Nucl Med Mol Imaging 2008;35:81520.

18. Tarkin JM, Joshi FR, Evans NR, et al. Detection of atherosclerotic Inflammation by (68)Ga-DOTATATE PET compared to [(18)F]FDG PET imaging. J Am Coll Cardiol 2017;69:1774-91. 